\title{
A comparison between model calculations and observations of sunspot oscillations
}

\author{
I. Rüedi ${ }^{1}$ and P. S. Cally ${ }^{2}$ \\ 1 Physikalisch-Meteorologisches Observatorium Davos/World Radiation Center, Dorfstr. 33, 7260 Davos Dorf, Switzerland \\ ${ }^{2}$ Centre for Stellar and Planetary Astrophysics, School of Mathematical Sciences, Monash University, Victoria 3800, Australia
}

Received 10 June 2003 / Accepted 25 August 2003

\begin{abstract}
We investigate the signal which is expected to be produced by magnetic field oscillations in sunspots umbrae due to the combination of the oscillation model, radiative transfer and observing procedure. For this purpose we investigate the signal expected to be produced by theoretical models of sunspot oscillations and compare them with the signal seen in observed power spectra of sunspot magnetograms. We show that the amplitudes of the observed oscillations are compatible with the predictions of the theoretical model of magnetoacoustic oscillations for the 5-min as well as for the 3-min band. For the particular sunspot umbral oscillation models used, our analysis suggests that most of the expected observed power in the magnetogram signal oscillations is actually due to cross-talk from the temperature and density oscillations associated with the magnetoacoustic wave. A detailed modelling of the observing procedure turns out to be of central importance for the assignment of the observed oscillations to a specific wave type.
\end{abstract}

Key words. Sun: activity - Sun: magnetic fields - Sun: oscillations - Sun: photosphere - Sunspots

\section{Introduction}

Sunspot velocity oscillations have been known for a long time. Relative to the quiet sun, sunspots harbor enhanced 3-min chromospheric oscillations and reduced 5-min photospheric oscillations (e.g. Lites 1992). Until recently no conclusive observations of oscillations of the sunspot magnetic field existed. However, in the past few years different groups have reported on such observations (Horn et al. 1997; Lites et al. 1998; Norton et al. 1998, 1999, 2001; Rüedi et al. 1998; Rüedi \& Solanki 1999; Bellot Rubio et al. 2000; Kupke et al. 2000; Settele et al. 2002b; Balthasar 2003). For a review on sunspot oscillations, see Staude (1999).

In general, models of (magnetoacoustic) sunspot umbral oscillations tend to predict far smaller amplitudes of oscillations of the magnetic field (relative to the observed amplitudes of the velocity oscillations) than are observed by most groups (Lites et al. 1998) and it is unclear if they are in agreement with the observations.

Earlier investigations (Rüedi et al. 1998; Settele et al. 2002a) focussed on the cross-talk produced by velocity oscillations into the magnetogram signal measured using the Michelson Doppler Interferometer (MDI, Scherrer 1995) using a static constant magnetic field. The results of Settele et al. (2002a), who take into account the fact that the measurements of MDI's different filters used to produce the magnetograms are not made strictly simultaneously, suggest that $2 / 3$ of the

Send offprint requests to: I. Rüedi, e-mail: i .ruedi@pmodwrc.ch 5-min signal seen in the magnetograms could have its origin in cross-talk.

Our aim is to investigate if the observational signature expected to be produced by present theoretical sunspot umbral oscillation models is compatible with the observations. Therefore, we will not review the subject of sunspot oscillations, but will concentrate on the comparison of a set of such observations with the observational signature expected from the theoretical umbral sunspot-oscillations model of Cally and co-workers (Cally et al. 1994; Lites et al. 1998) and on the physical origin of the expected signal. Preliminary results of this work have been published by Rüedi et al. (1999).

We first recall the observations on which we base our work (Sect. 2) and describe the model (Sect. 3). In Sect. 4 we determine the expected observational signature produced by the model and filtergram measurements and investigate the origin of the produced signal in Sect. 5. Section 6 addresses another measurement method - the center-of-gravity method. The case of 3-min oscillations is briefly looked at in Sect. 7. Finally the results are discussed in Sect. 8.

\section{Observational signature}

The observations on which we base our comparison have been presented by Rüedi et al. (1998). These are MDI high resolution field of view magnetograms and velocitygrams. More specifically, we concentrate on the power spectra they observed at the position with strongest magnetic field in the largest 
sunspot of the region NOAA 7999 on Nov. 27 1996. This sunspot was located very close to disc center.

The power integrated over the frequency range 2.6$3.9 \mathrm{mHz}$ corresponds to rms fluctuations of $6.4 \mathrm{G}$ and $76 \mathrm{~m} \mathrm{~s}^{-1}$ for the magnetogram and velocity signal respectively. The magnetogram oscillations lead the velocity oscillations by approximately $65^{\circ}$. These are the observational results, which we now try to reproduce using a theoretical sunspot-oscillations model. For more clarity these values are summarized in Table 1.

The following spot of the group shows peaks in the $6 \mathrm{mHz}$ band which will also be briefly investigated in Sect. 7. These observations have the advantage to show power located in the central part of umbrae which strongly reduces the risk of any contamination by stray-light from the penumbra or evolution of umbra-penumbra boundary.

Table 1. Summary of observations.

\begin{tabular}{lccc}
\hline \hline Frequency $[\mathrm{mHz}]$ & $\delta B_{\mathrm{rms}}[\mathrm{G}]$ & $\delta v_{\mathrm{rms}}\left[\mathrm{m} \mathrm{s}^{-1}\right]$ & Phase $\left[^{\circ}\right]$ \\
\hline $2.6-3.9$ & 6.4 & 76 & 65 \\
\hline
\end{tabular}

\section{The model}

As input for our computation, we use the fluctuations produced by a model of sunspot umbral oscillations. These fluctuations are then superimposed on a sunspot model atmosphere. Radiative transfer calculations are carried out through this structure. Finally the observing procedure is applied to the resulting spectral line profiles. More details on each stage of the computations are given below.

\subsection{Model of sunspot oscillations}

The model used assumes a monolithic structure of the umbral magnetic field. It consists of a uniform vertical background magnetic field of $3000 \mathrm{G}$ in hydrostatic balance. The temperature structure of the model consists of 3 layers: in the photosphere and chromosphere (between $z=-122 \mathrm{~km}$ and $z=2146 \mathrm{~km}, z=0 \mathrm{~km}$ being the solar surface, i.e. the layer with $\tau=1$ ) it follows the Malty $\mathrm{M}$ sunspot model (Maltby et al. 1986). Below $z=-122 \mathrm{~km}$, it is described by an isentropic polytrope. Finally, it possess an isothermal $\left(2 \times 10^{6} \mathrm{~K}\right)$ corona above $z=2146 \mathrm{~km}$. Linear adiabatic waves are computed in this structure for distinct oscillation frequencies and horizontal wavenumbers. For more information on the model (see Cally \& Bogdan 1993; Cally et al. 1994; Lites et al. 1998).

The case of umbral oscillations in a spreading magnetic field was discussed at length by Cally (1983). Under certain simplifying assumptions, it was found that magnetoacoustic waves are not substantially altered by the field spread (though torsional Alfven waves are). Nevertheless, the precise effect of declining field strength with height in the context of assessing the importance of cross-talk to observations of magnetic perturbations on sunspot atmospheres deserves further study.
Here we concentrate on one of the oscillatory modes of the umbra with a period of $5 \mathrm{~min}$ and horizontal wavenumber $1.96 \times 10^{-3}+i 6.18 \times 10^{-5} \mathrm{~km}^{-1}$ because it is a 5 -min band mode producing rather large $B$-oscillations relative to its $v$-oscillations.

The output of the sunspot-oscillations code consists of the (complex) perturbations of the magnetic field vector, velocity, density and temperature as a function of height in the atmosphere. For test purposes (cf. Sect. 5) we have in some cases retained only some of the perturbations for the further computations and set the others to 0 . This will help us to see how each of them indirectly affects the measured magnetogram and velocitygram through small changes of the line profile.

We computed these perturbations for a series of time-steps sampling the $300 \mathrm{~s}$ period of the wave. These perturbations are then superposed on the Maltby $\mathrm{M}$ atmospheric model appropriate for a sunspot umbra and permeated by a constant magnetic field of $1880 \mathrm{G}$ corresponding to that of the observed sunspot. Radiative transfer calculations through this structure yield the emerging spectral line profiles.

\subsection{Simulation of MDl's filters}

MDI magnetograms and velocitygrams are computed from images obtained using 4 filters, which sample the Ni I $6768 \AA$ spectral line at different wavelengths (Scherrer et al. 1995). A combination of those 4 readings deliver a value which can be converted to velocity using a look-up table. The magnetogram signal is proportional to the difference of the velocity values observed in right and left circularly polarized light.

In order to compare the observations with the spectral line profiles produced by the radiative transfer model, we apply MDI's filter functions on each of the computed line profiles and determine the corresponding synthetic magnetogram and velocitygram signal therefrom.

In our computation, we assume that all filter measurements are carried out simultaneously. This is not absolutely correct as it effectively takes $30 \mathrm{~s}$ to carry out the observations (Settele et al. 2002a), but it has the advantage of enabling us to isolate the effects of the various physical parameters which influence the measured signals. It does however miss some of the crosstalk from the velocity to the magnetogram time series.

\section{Comparison of observational and theoretically expected signatures}

\subsection{Constant magnetic field}

Figure 1 shows the results of the computations described above as a function of time (for one period of the wave). The upper (lower) panel depicts the magnetogram (velocity) fluctuations around their mean values, expected to be seen by MDI for the model under consideration. The oscillations are linear and have been scaled so that $\delta v_{\text {rms }} \approx 75 \mathrm{~m} \mathrm{~s}^{-1}$. This corresponds to the amplitude of the velocity oscillations observed in the 5-min band by Rüedi et al. (1998). We can now compare the corresponding synthetic magnetogram signal with that present in the observations. The former turns out to show significant 

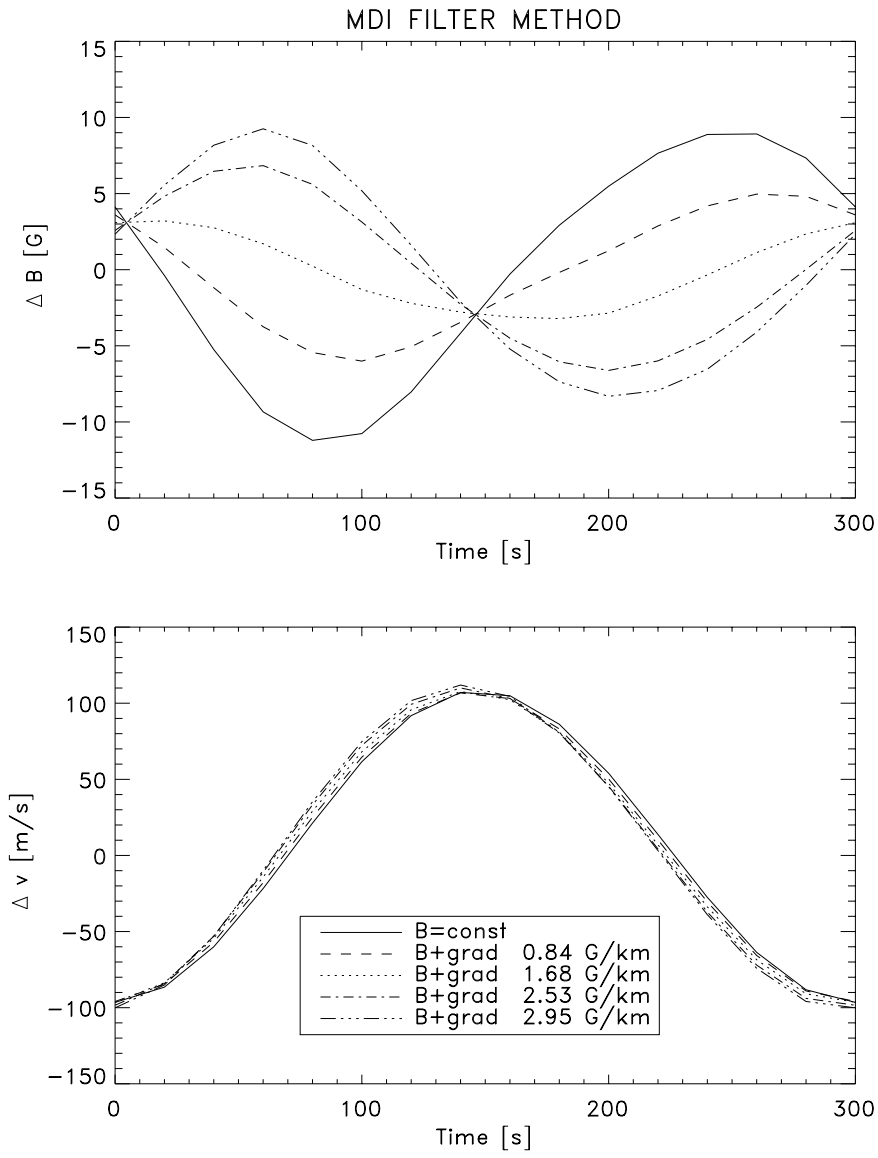

Fig. 1. Deviation of the magnetogram signal (top) and Doppler velocity shift from their mean values as a function of time over one period of the wave. The solid curves represent the case of a constant vertical magnetic field, while the other curves show the results obtained by adding a vertical magnetic field gradient into the atmospheric model. The magnitude of the gradient is indicated in the lower figure.

oscillations with a rms amplitude of $7.2 \mathrm{G}$. In principle, this is large enough to reproduce the magnitude of the observations. However, there is a discrepancy in the phase. Figure 1 shows clearly that in this case, the synthetic velocity leads the magnetogram signal which contradicts the observations.

Figure 2 shows the perturbations which were implemented in the model atmosphere at the height of $98 \mathrm{~km}$, within the height of formation of the line. It can be seen that the amplitude of the velocity perturbations correspond to the observed one, while the magnetic field perturbations are much smaller in the model at this given height then those detected taking into account the radiative transfer and detection scheme of MDI.

\subsection{Effect of a magnetic field gradient}

Numerous observations show that the magnetic field strength in sunspots decreases with height (e.g. Wittmann 1974; Balthasar \& Schmidt 1993; Bruls et al. 1995; Rüedi et al. 1995; see Solanki 2003 for a review). This gradient is strongest in the photosphere and appears to decrease with height. In the presence of such a gradient, the influence of the MHD wave on the line profile (or measured magnetogram signal) may be twofold. Firstly, there is a contribution due to the time dependence of

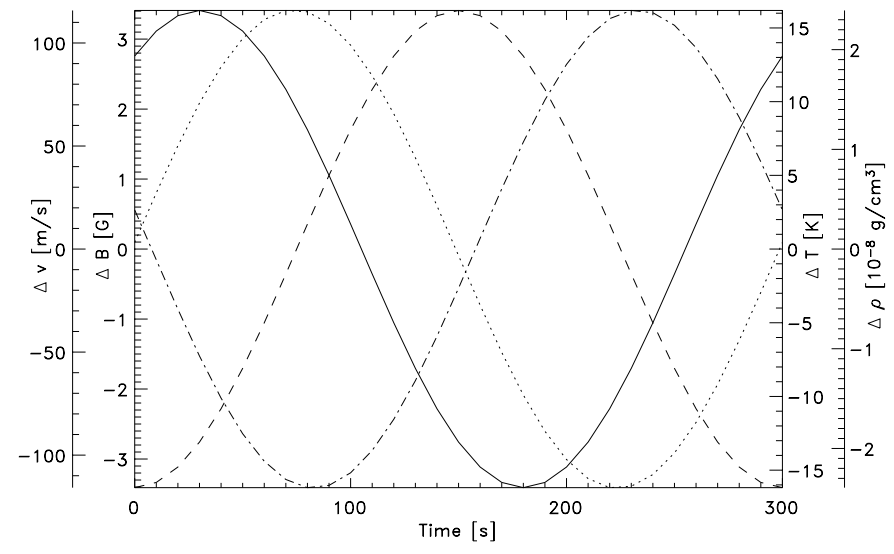

Fig. 2. Perturbations produced by the model at a height of $98 \mathrm{~km}$ in the sunspot atmosphere. The lines correspond to the following parameters: - solid: vertical magnetic field - dashed: vertical velocity - dot-dashed: temperature - dotted: density.

the magnetic field strength at a specific height. Secondly, the temperature and density oscillations inherent to the wave also cause the height of formation of the spectral line in the atmosphere to oscillate slightly. Due to the general decrease of the magnetic field strength with height, this would translate into a change in the sampled magnetic field strength.

Unfortunately, the present state of the theoretical models used here does not allow us to self consistently compute waves in an atmosphere with height-dependent magnetic field. To circumvent this we use the perturbations of the atmospheric parameters computed in the presence of a constant magnetic field and implement them into the Maltby $M$ model to which we add a magnetic field gradient. The gradient is fixed at $\log \tau=$ -1.05 . It is our hope that a magnetic field gradient does not affect the perturbations over the height range over which the line is formed too strongly. The results of these computations are included in Fig. 1 for different values of the magnetic field gradient. None of these gradients exceeds the observed gradients typically reported in the literature (a review is given by Solanki 2003). As can be seen from the figure, the influence of an increasing magnetic field gradient is to reduce the amplitude of the observed magnetic field fluctuations measured by the magnetograph and eventually to invert its sign. In the process, the sign of the phase difference between the velocity and magnetogram signal fluctuations changes and the $B$-oscillations seen in the magnetogram start leading over the velocity fluctuations. This agrees with the behavior seen in the observations. It therefore turns out that the amplitude of the observed magnetogram and velocity oscillations can be reproduced by the model simultaneously with their phase relationship if a magnetic field gradient of roughly $-3 \mathrm{G} / \mathrm{km}$ is present at the analyzed location in the sunspot atmosphere.

\section{Origin of the observed magnetogram fluctuations}

Astonishingly, the fluctuations of the magnetic field strength present in the model atmosphere at a constant height (located inside the range of height of formation of the spectral line) which are shown in Fig. 2 are significantly smaller than the 
values suggested by the amplitude of the solid curve in Fig. 1. For a measured rms velocity fluctuation of $75 \mathrm{~m} \mathrm{~s}^{-1}$, the corresponding synthetically observed magnetic fluctuations correspond to 7.2 G rms. However, at a specific height in the atmospheric model (within the height of formation of the line), the magnetic field strength fluctuates by no more than $2.5 \mathrm{G} \mathrm{rms,}$ as can be seen in Fig. 2. (Note that since the magnetic field is principally vertical, $|\delta \boldsymbol{B}|$ is effectively $\delta B_{z}$ ). In this section we try to understand what is at the origin of the magnetogram signal oscillations in spite of the small intrinsic magnetic field oscillations.

For that purpose, we investigate how each of the physical parameters taking part in the oscillations affects the spectral line profiles and consequently the output of MDI's filters. As already mentioned, the perturbations which we implemented into the atmospheric model are those of velocity, magnetic field, temperature and density. In the following analysis we take a step-by-step approach and switch off the perturbations in one quantity after another in each step. For this detail investigation we consider the case of an atmospheric model with a constant vertical magnetic field.

In a first step, we implemented all the fluctuations supplied by the oscillations model, except the magnetic field fluctuations. This case is represented by the solid curves in Fig. 3. It can be seen that although the magnetic field perturbations have been neglected, the amplitude of the synthetic magnetogram signal is similar to the magnitude when these perturbations are taken into account (solid curves of Fig. 1). In order to simplify the understanding of Fig. 3, the magnetic field fluctuations have been neglected for all the plotted curves.

In a second step, we also removed the density perturbations (dashed curves). The amplitude of the expected measured oscillations is reduced by a factor of $1 / 3$. This means that a significant cross-talk from the density perturbations into the magnetogram signal is present.

In a third step, we retained the density perturbations, but neglected the temperature perturbations (dotted curves). Here again, a strong reduction of the expected measured oscillations is achieved and reaches almost a factor of $2 / 3$. Consequently, if both the density and temperature perturbations are neglected, i.e. if only the velocity perturbations are implemented, almost no magnetogram oscillation is expected to be measured, as shown by the dash-dotted line in Fig. 3.

In summary, most of the measured magnetogram oscillations produced by this particular wave are actually due to crosstalks from the density and temperature oscillations (coupled with the velocity oscillations) and only a small amount is due to true magnetic field oscillations. This is partly a peculiarity of MDI's sensitivity to changes of the spectral line profile shape, which are mostly due to the temperature and density variations rather then to the magnetic fields and velocity variations inherent to the wave.

\section{Center of gravity method}

The observational signature to be expected from magnetoacoustic gravity waves depends strongly on the type of observations and on the method used to determine the magnetic field
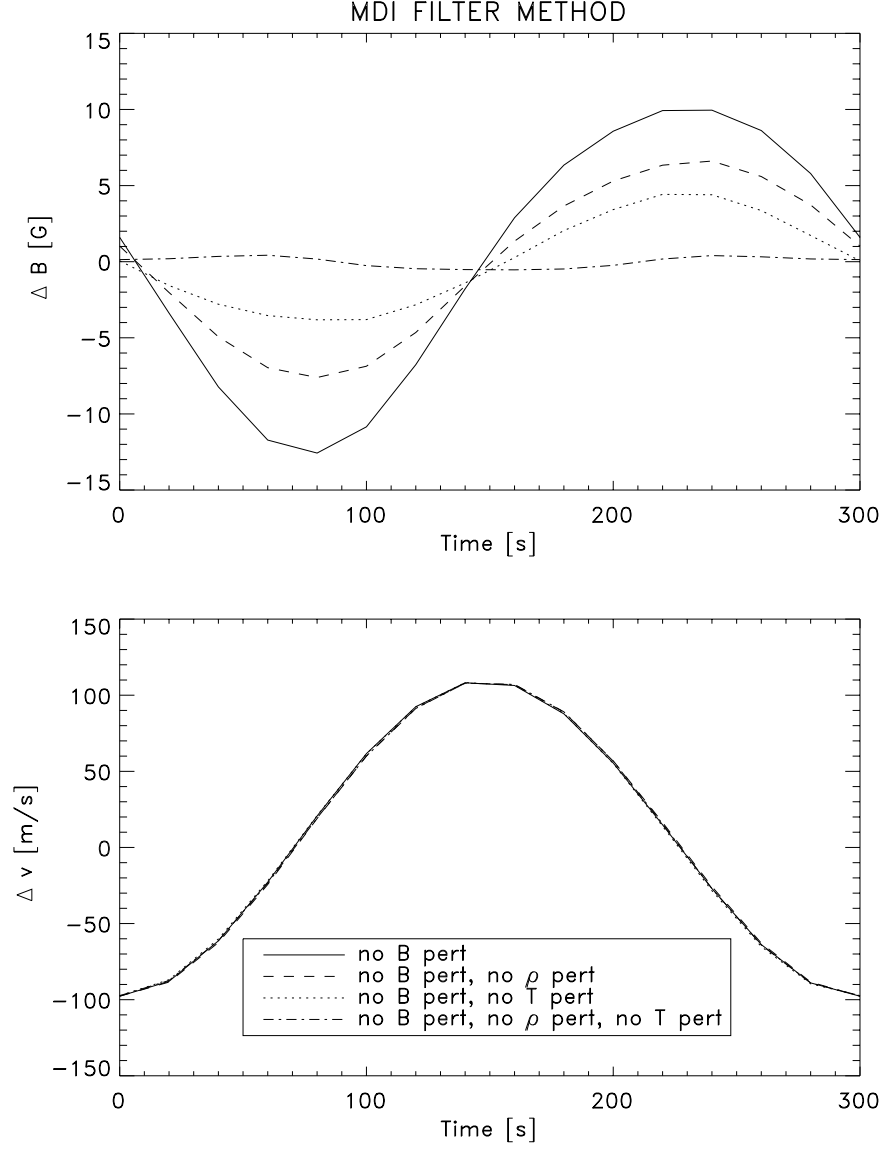

Fig. 3. Same as Fig. 1, but all the curves now refer to an atmospheric model with a constant vertical magnetic field. Also in all cases the magnetic field perturbations produced by the wave have been neglected. In addition, the following perturbations have been neglected: - dashed curve: density perturbations - dotted curve: temperature perturbations - dot-dashed curve: density and temperature perturbations.

strength and Doppler velocity shift (Settele et al. 2002a). The results presented so far described filter observations. Figure 4 is similar to Fig. 1 but shows the signature which would be expected using spectroscopic observations of Stokes $I$ and $V$ and determining the magnetic field strength using the centerof-gravity method (using of course the same spectral line as used by MDI: Ni I $6768.78 \AA$ ). It can be seen that the presence of a magnetic field gradient has a similar effect, but the relative phase between the magnetic field and velocity signals obtained from the evaluation has the opposite sign to that obtained with MDI. This illustrates that it is far from trivial to determine types of oscillations simply from the phase shift between the measured magnetic and velocity signal. Attempting to associate a specific wave type to observations consequently has to include a precise modelling of the observing and analysis procedure.

This difference between the 2 methods may be understood by keeping in mind that the velocity and magnetic field delivered by the magnetogram as well as the center-of-gravity method do not correspond to values at a specific height in the solar atmosphere, but rather give an approximation of those values over the height of formation of the spectral line (Uitenbroek 2003). In addition, the methods are differently sensitive to 

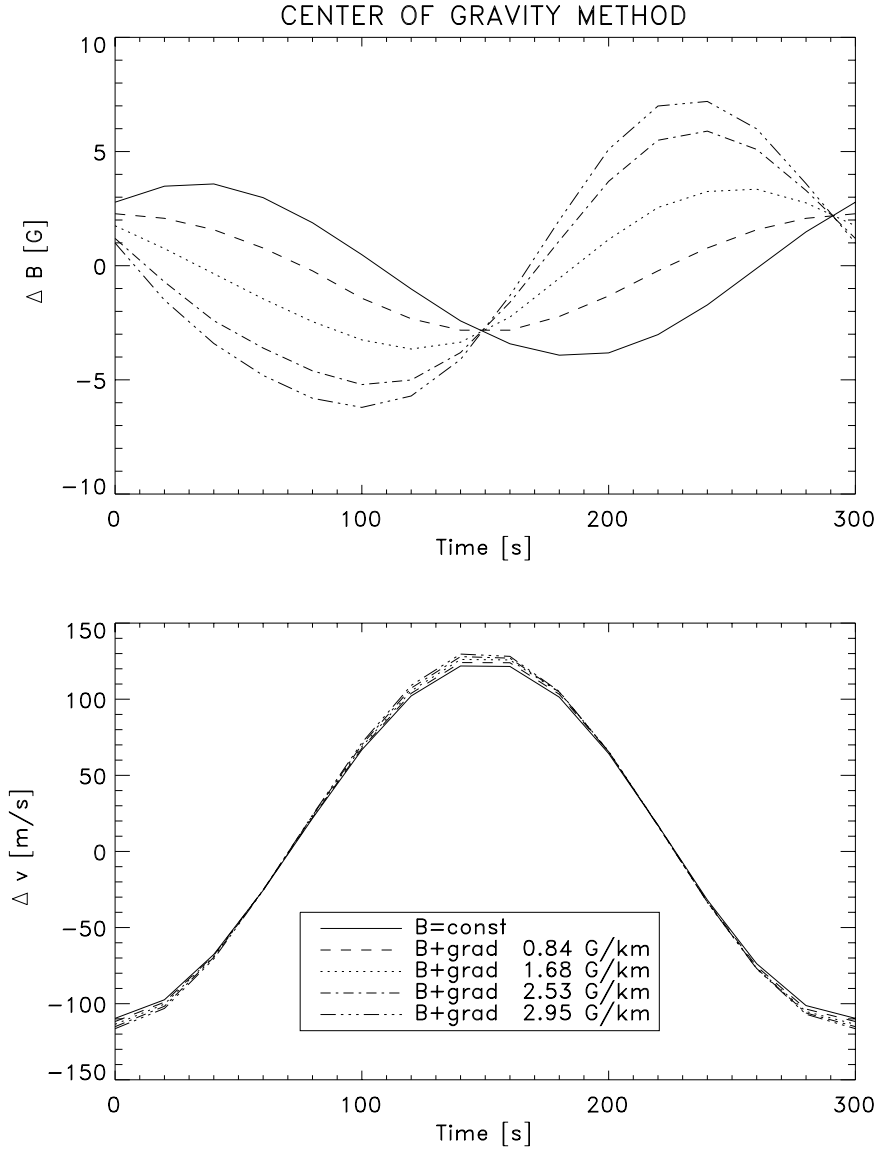

Fig. 4. Same as Fig. 1, but now simulating observations of Stokes $I$ and $V$ measured with a spectrograph and using the center-of-gravity method to determine the magnetic field strength.

subtle changes of the line profile's shape. Inversion techniques may solve this problem by providing the depth-dependence of the atmospheric parameters. However, even inversion techniques are confronted with the difficulty to relate the optical depth with the geometrical height (Bellot Rubio et al. 2000).

It should also be stressed again that in this paper we assume that all the measurements necessary to determine the velocity and magnetic field at one time-step were carried out simultaneously. In addition, the time necessary to carry out the observation will also produce some additional cross-talk as was shown by Settele et al. (2002a).

\section{3-min oscillations}

The observations of Rüedi et al. (1998) also show a sunspot with a power peak in the 3-min band. The signals integrated over that band correspond to oscillations of $5.4 \mathrm{G} \mathrm{rms}$ and $10.7 \mathrm{~m} \mathrm{~s}^{-1} \mathrm{rms}$. In this case the magnetic oscillation is much larger relative to the velocity oscillations than in the 5-min band. We are interested to see if the amplitudes of such oscillations also correspond with the predictions from models. For that purpose we used 3 oscillatory modes resulting from Cally's model with periods of respectively $179.4 \mathrm{~s}, 153.8 \mathrm{~s}$ and $151.6 \mathrm{~s}$. We scaled the perturbations so that the order of magnitude of the simulation's output corresponds to that of the observed rms velocity oscillations. The results are listed in Table 2 for the different modes.
Table 2. 3-min oscillations.

\begin{tabular}{lccc}
\hline \hline Period $[\mathrm{s}]$ & $\delta B_{\mathrm{rms}}[\mathrm{G}]$ & $\delta v_{\mathrm{rms}}\left[\mathrm{m} \mathrm{s}^{-1}\right]$ & $\delta B_{\mathrm{rms}} / \delta v_{\mathrm{rms}}[\mathrm{Gs} / \mathrm{m}]$ \\
\hline 179.4 & 2.1 & 15.5 & 0.1 \\
153.8 & 5.6 & 4.7 & 1.2 \\
151.6 & 6.0 & 4.2 & 1.4 \\
Observed & 5.4 & 10.7 & 0.5 \\
\hline
\end{tabular}

It can be seen that depending on the mode considered the ratio of the magnetic to the velocity oscillations is either larger or smaller than the observed ratio. This suggests that 3-min magneto-acoustic modes are also able to reproduce the observations. Our aim being to show that the state-of-the-art model calculations are in principle able to reproduce the observations, we do not attempt to pick out the exact mode which reproduces these observations the best. It should be noted that Settele et al. (2002a) were unable by far to reproduce the amplitude of the 3-min magnetogram fluctuations simply by cross-talk from the velocity signal (using a static constant magnetic field).

\section{Discussion}

Different types of oscillations imply different relationships between the magnetic field and velocity oscillations. Ulrich (1996) clearly summarized the $v-B$ relation for Alfvén waves and compressional waves. The Alfvén waves are expected to be observable away from disc center and the phase angle between $\delta B$ and $\delta v$ should be 0 . The compressional mechanism is best observed close to disc center and the phase angle between $\delta B$ and $\delta v$ should be close to $\pi / 2$ with an additional random component, whereby the downward velocity is expected to lag behind the magnetic field oscillations.

Sunspots are not expected to harbor strong oscillations of the magnetic field strength. However, various groups reported on the observations of such oscillations. They reached different conclusions as far as the origin of the oscillations is concerned. Horn et al. (1997) presented a significant detection, but didn't analyze it further. Lites et al. (1998) claim that their detection is most probably due to instrumental cross-talk. They based their reflection on the oscillations expected in a theoretical model at a specific height and on the phase relation between the magnetic and velocity oscillations. Norton et al. (1998) interpreted their observations in terms of magnetoacoustic waves due to their phase relationship. Rüedi et al. (1998) came to the same conclusion using a similar argument and doing simple tests on the possible influence of some atmospheric parameters. Bellot Rubio et al. (2000) see the origin of the oscillations they measure in opacity effects.

Here we have shown that a theoretical model of sunspot umbral oscillations is able to reproduce the 5-min oscillations (amplitudes and relative phase) observed by Rüedi et al. (1998) if a magnetic field gradient of the order of $3 \mathrm{G} / \mathrm{km}$ is present in the atmospheric model. We showed that a large part of the measured magnetic oscillations is actually due to cross-talk from the density and temperature fluctuations produced by the wave itself. This strengthens the long-held view that weak 
magneto-atmospheric waves are indeed present in sunspot umbrae. The amplitude of the observations carried out in the 3-min band are also compatible with such models. This analysis doesn't rule out other interpretations of the observations, but shows that they are not in contradiction with the presently existing theoretical models of sunspot umbral observations, in spite of the small magnetic fluctuations that these models exhibit at the height of the line formation.

Finally, our analysis demonstrates that in order to properly interpret the nature of the observed oscillations, it is absolutely necessary to directly compare the observations with a model taking into account the physical properties of the oscillation model, coupled with radiative transfer calculations as well as taking into account the complete observing and analysis procedure. In other words it is not sufficient to compare the measured velocity and magnetic signals with the model's characteristic signatures as for example the phase difference between velocity and magnetic field fluctuations expected at a definite height for a certain oscillation type, but all the details of the radiative transfer and observing procedure have also to be taken into account if one wants to associate the observations with a specific oscillation type. This is in agreement with the paper by Settele et al. (2002a) who address the influence of the duration of the observing procedure on the produced magnetogram oscillations.

MDI observations clearly show the presence of MHD waves in sunspot umbra which are shown to be compatible with the present theoretical models of sunspot umbral oscillations. However, the informational content of MDI's measurements do not give enough information to fully characterize the physical state of the umbra which is necessary to uniquely associate the observations with a specific oscillation type.

Ways to circumvent this problem are for example simultaneous observations with other observing systems delivering additional information on the physical state of the umbra such as possibly magnetograms sampled in other spectral lines, i.e. other height in the atmosphere, or the use of spectro-polarimetric observations as presented by Bellot Rubio et al. (2000) and further analyzed by Khomenko et al. (2003). However, such ground-based observations have the shortcomings of being affected by seeing fluctuations and produce only 1-D spatial cuts through the sunspot in contrast to the 2-D maps produced by MDI.

Acknowledgements. The authors would like to thank T. Bogdan and S. Solanki for their support and helpful comments and the latter for carefully reading and commenting on the manuscript. SOHO is a cooperative mission between the ESA and NASA. This work was partly supported by the Swiss National Science Foundation, grant No. 21-45083.95, and by a grant from the ETH Zürich.

\section{References}

Balthasar, H. 2003, Sol. Phys., in press

Balthasar, H., \& Schmidt, W. 1993, A\&A, 279, 243

Bellot Rubio, L. R., Collados, M., Ruiz Cobo, B., \& Rodriguez Hidalgo, I. 2000, ApJ, 534, 989

Bruls, J. H. M. J., Solanki, S. K., Carlsson, M., \& Rutten, R. J. 1995, A\&A, 293, 225

Cally, P. S. 1983, Sol. Phys., 88, 77

Cally, P. S., \& Bogdan, T. J. 1993, ApJ, 402, 721

Cally, P. S., Bogdan, T. J., \& Zweibel, E. G. 1994, ApJ, 437, 505

Horn, T., Staude, J., \& Landgraf, V. 1997, Sol. Phys., 172, 69

Khomenko, E. V., Collados, M., \& Bellot Rubio, L. R. 2003, ApJ, 588, 606

Kupke, R., Labonte, B. J., \& Mickey, D. L. 2000, Sol. Phys., 191, 97

Lites, B. W. 1992, in Sunspots: Theory and Observations, ed. J. H. Thomas, \& N. O. Weiss (Dordrecht: Kluwer), 261

Lites, B. W., Thomas, J. H., Bogdan, T. J., \& Cally, P. S. 1998, ApJ, 497, 464

Maltby, P., Avrett, E. H., \& Carlsson, M., et al. 1986, ApJ, 306, 284

Norton, A. A., Ulrich, R. K., Bogart, R. S., Bush, R. I., \& Hoeksema, J. T. 1998, in New Eyes to See Inside the Sun and Stars, ed. F.-L. Deubner, J. Christensen-Dalsgaard, \& D. Kurtz (Dordrecht: Kluwer), IAU Symp., 185, 453

Norton, A. A., Ulrich, R. K., Bush, R. I., \& Tarbell, T. 1999, in 3rd Advances in Solar Physics Euroconference, Solar Magnetic Fields and Oscillations, ed. B. Schmieder, A. Hofmann, \& J. Staude, ASP Conf. Ser., 136

Norton, A. A., Ulrich, R. K., \& Liu, Y. 2001, ApJ, 561, 435

Rüedi, I., Solanki, S. K., \& Livingston, W. 1995, A\&A, 293, 252

Rüedi, I., Solanki, S. K., Stenflo, J. O., Tarbell, T., \& Scherrer, P. H. 1998, A\&A, 335, L97

Rüedi, I., \& Solanki, S. K. 1999, in 3rd Advances in Solar Physics Euroconference, Solar Magnetic Fields and Oscillations, ed. B. Schmieder, A. Hofmann, \& J. Staude, ASP Conf. Ser., 131

Scherrer, P. H., Bogart, R. S., \& Bush, R. I., et al. 1995, Sol. Phys., 162,129

Solanki, S. K. 2003, A\&ARv, 11, 153

Settele, A., Carroll, T. A., Nickelt, I., \& Norton, A. A. 2002a, A\&A, 386, 1123

Settele, A., Sigwarth, M., \& Muglach, K. 2002b, A\&A, 392, 1095

Staude, J. 1999, in 3rd Advances in Solar Physics Euroconference, Solar Magnetic Fields and Oscillations, ed. B. Schmieder, A. Hofmann, \& J. Staude, ASP Conf. Ser., 113

Uitenbroek, H. 2003, ApJ, 592, 1225

Ulrich, R. K. 1996, ApJ, 465, 436

Wittmann, A. D. 1974, Sol. Phys., 36, 29 